\title{
EL PAGO POR SERVICIOS AMBIENTALES COMO ALTERNATIVA PARA EL USO SOSTENIBLE DE LOS SERVICIOS ECOSISTÉMICOS DE LOS PÁRAMOS
}

\section{Payment for Environmental Services as an alternative for the sustainable use of peatland ecosystem services}

\author{
JHOHNNY ROJAS P 1
}

1Universidad del Valle

E-mail: johnny.harold.rojas@correounivalle.edu.co

Recibido: 11 de Agostode 2010

Aceptado: 11 de Abril de 2011

\section{Resumen}

Los páramos proporcionan múltiples servicios ecosistémicos (SE), como la conservación de la biodiversidad, la captura de carbono, la oferta de hábitats para especies, la regulación climática y la regulación hídrica. La degradación de los páramos debido al cambio climático, pero principalmente por la intensificación de la agricultura y la ganadería en los últimos 50 años, genera la perdida de estos SE. Posiblemente, los Pagos por Servicios Ambientales (PSE) ofrecen oportunidades, pero tienen grandes limitaciones, para lograr un uso sostenible de los SE ofrecidos por los páramos por parte de comunidades locales que realizan prácticas agrícola y ganaderas en este ecosistema.

Palabras claves: Páramos, Pago por servicios ambientales, Servicios ecosistémicos, Uso sostenible.

\section{Abstract}

The peatlands provide diverse ecosystem services (ES) such as biodiversity conservation, carbon sequestration, the supply of habitats to species, and the climate and water regulation. Peatlands degradation due to climate change but mainly because of agriculture and livestock intensification in the last fifty years, result in the lost of these ES. Probably, Payment for Ecosystem Services schemes offer an opportunity, but also have great limitations, to reach a sustainable use of the ES provide by peatlands in the case of local communities that carry out agricultural and livestock activities on this ecosystem.

Keywords: Peatlands, Payment for environmental services, Ecosystem services, Sustainable use. 


\section{INTRODUCCIÓN}

Los páramos colombianos son reconocidos por su oferta de servicios ecosistémicos (SE) claves para el bienestar humano, como la conservación de la biodiversidad, la provisión de hábitats para distintas especies (Rangel 2002), la regulación hídrica, la absorción de carbono (Buytaert et al. 2007) y servicios culturales asociados con el uso de estos ecosistemas para actividades como el turismo, la investigación e incluso el disfrute espiritual.

La realidad es que los páramos en Colombia vienen siendo altamente afectados e incluso existe una visión bastante negativa sobre la irreversibilidad de los procesos de transformación y destrucción de estos biomas (Procuraduría General de la Nación 2007). Las dos causas fundamentales del proceso de degradación de los páramos son el cambio climático (impacto global), pero fundamentalmente las diversas actividades antrópicas que en estos ecosistemas se realizan (impacto local) (Hofstede 2002).

La agricultura y la ganadería, actividades que se desarrollan en los páramos hace por lo menos 3.000 años A.P. (Hofstede et al. 1988), se han intensificado en los últimos 50 años (Reyes 1996), generando importantes cambios en el uso del suelo paramuno, debido a sus prácticas de producción poco limpias. Esto conlleva la pérdida y/o alteración de los SE ofrecidos por los páramos, es decir un uso no sostenible de los mismos.

Los Pagos por Servicios Ambientales (PSA) se han propuesto como una herramienta óptima que puede contribuir a la conservación de los $\mathrm{SE}$, al generar incentivos económicos para que quienes los usan de manera insostenible realicen prácticas productivas más limpias (o en caso extremo no las realicen), manteniendo la oferta de SE. Existen sin embargo múltiples problemas por los cuales este esquema parece poco efectivo para cumplir su propósito.

El objetivo de este artículo es analizar si los PSA son una opción efectiva para la conservación de los SE que ofrecen los páramos. Para esto, a continuación el artículo presenta de manera breve el enfoque, tipología y oferta de SE de los páramos. Luego se discute el problema de degradación de los páramos debido a la agricultura y la ganadería y la pérdida de SE asociado a estas actividades; finalmente, se presentan algunas bases conceptuales del esquema PSA y se analiza su efectividad para preservar los SE de los páramos.

\section{DESCRIPCIÓN}

\section{Servicios ecosistémicos: antecedentes, definiciones y tipologías}

Existe algún consenso en cuanto a que el término servicios ecosistémicos fue utilizado por primera vez a inicios de los 80's por parte de Erlich \& Erlich (Gomez-Baggethum et al. 2010, Peterson et al. 2010). Estos investigadores proponían que las funciones naturales de los ecosistemas podían ser vistas como "servicios" que estos le prestaban a los seres humanos, buscando con ello establecer que la conservación de la biodiversidad era necesaria para poder mantener los servicios de los ecosistemas y no poner en riesgo la vida de los seres humanos. Tal como lo señala Peterson et al. (2010). "el concepto de servicios ecosistémicos se usó para enseñar a la gente que los ecosistemas nos prestan servicios, demostrando entonces el valor de las funciones ecosistémicas para la humanidad".

Sin embargo, esta intención netamente pedagógica fue rápidamente superada durante los 90`s por la prioridad que se le dio a la valoración monetaria de los SE, impulsada fundamentalmente por la economía ambiental. Esto se debió en parte, a que los conservacionistas vieron en la valoración económica otra forma para ayudar a demostrar la importancia de los servicios ecosistémicos. Uno de los estudios más representativos de la valoración monetaria de la naturaleza se produjo cuando Constanza et al. (1997) valoraron $17 \mathrm{SE}$ asociados a 16 biomas de todo el planeta, resultando en que la contribución de tales servicios estaba en el rango entre los US 16-54 trillones anuales, con un promedio de US 33 trillones.

Existen diversas definiciones para los SE Daily (1997) entiende que los servicios ecosistémicos son las condiciones y procesos a través de los cuales los ecosistemas naturales y las especies que los conforman, sostienen y le dan sentido a la vida humana. Por su parte, Evaluación de los Ecosistemas del Milenio (2003) define los SE como los beneficios que la gente obtiene de los ecosistemas. Esta definición, aunque criticada por ser muy escueta, ha tenido una alta relevancia pues posicionó y le otorgó una alta prioridad al tema de los servicios ecosistémicos en la agenda internacional. Finalmente, el concepto de servicios ecosistémicos que se considera más relevante es el formulado por (Fisher et al. 2009), quienes afirman que los servicios ecosistémicos son todos los componentes de los ecosistemas que se utilizan (activa o pasivamente) para producir bienestar humano.

La clasificación y medición de los SE cuenta con varias tipologías. Una de ellas es la proporcionada por la Evaluación de Ecosistemas de Milenio-EEM (2003), que es de las más 
representativas y utilizadas. Sin embargo, la clasificación de De Groot et al. (2002), ofrece un esquema comprensivo para clasificar, evaluar y valorar los servicios ecosistémicos. Los autores citados proponen que a partir de la estructura y procesos naturales de los ecosistemas se producen cuatro funciones ecosistémicas:

- Regulación: se relaciona con la capacidad de los ecosistemas para regular los procesos ecológicos esenciales y los sistemas de soporte de vida, a través de los ciclos biogeoquímicos y otros procesos de la biosfera.

- Hábitat: proporcionar el espacio vital indispensable para la reproducción de plantas salvajes y especies animales.

- Producción: de manera resumida consiste en la provisión de recursos naturales. Dicho de manera más especializada se refiere a los procesos de fotosíntesis y asimilación de nutrientes por los autótrofos, quienes convierten la energía, el agua, los nutrientes y el dióxido de carbono en estructuras de carbohidratos que usan luego los productores secundarios para producir biomasa.

- Información: como la evolución humana tuvo lugar en hábitats no domesticados, los ecosistemas naturales proporcionan una "función de referencia" y contribuyen a la salud humana proporcionando oportunidades para la reflexión, el enriquecimiento espiritual, el desarrollo cognitivo, la recreación, etc.

De estas 4 funciones se derivan una lista de por lo menos 34 servicios ecosistémicos que se considera tienen un valor total, compuesto por su valor ecológico, socio-cultural y económico. El reconocimiento de este valor total, es entonces el que permite tomar decisiones en términos de opciones de política y mecanismos de gestión de los servicios ecosistémicos.

\section{Oferta de servicios ecosistémicos en páramos}

El páramo es un ecosistema complejo, en el que existe una rica simbiosis de múltiples atributos biofísicos y diversas expresiones socioculturales, que configuran la oferta de una variedad de SE. Los páramos solo existen en la zona andina de Latinoamérica y algunas áreas de Centroamérica (UICN 2003). Colombia cuenta con un $49 \%$ de los páramos del mundo en su territorio (Congreso Nacional de Páramos 2009), los cuales ocupaban una extensión de $14.434 \mathrm{~km} 2-1.3 \%$ del área continental del país- a inicios del siglo XXI (Ojeda et al. 2001).

La conservación de la biodiversidad es un SE relacionado con la variedad, pero especialmente el endemismo, de la vegetación y la fauna paramuna. Según Rangel-CH (2008) "la vegetación y la flora paramuna de Colombia es la más diversa y variada". Además, el $60 \%$ de las especies de plantas vasculares se encuentran en los páramos colombianos (Rangel-CH 2008); a la vez que la vegetación existente en ellos representa todas las combinaciones fitoecológicas de vegetación presentes en los páramos de otros países (Rangel 2002). En los páramos colombianos existen 130 especies de mariposas, 15 especies de reptiles y 87 de anfibios, ranas y sapos, 154 especies de aves -incluyendo algunas parejas de cóndores reintroducidos- y 70 especies de mamíferos (Instituto de Investigación de Recursos Biológicos Alexander von Humboldt 2007).

La interacción y variedad de formas físicas presentes en el páramo, como las colinas, depresiones, riachuelos, pantanos, crestas, etc., producen una gran diversidad de hábitats (Hofstede 2002), que le sirven de refugio a las especies de flora y fauna y se convierten en otro SE que proporciona este ecosistema.

Los páramos cumplen un rol fundamental en la regulación hídrica, catalogado como el principal SE que se deriva de estos ecosistemas (Ministerio de Medio Ambiente 2002). En Colombia, el abastecimiento de agua del $70 \%$ de los municipios depende de los páramos, donde nacen los principales ríos que recorren el país (Instituto de Investigación de Recursos Biológicos Alexander von Humboldt 2007). El potencial de regulación hídrica del páramo sed relaciona con su alta capacidad de retención de agua, la cual alcanza a ser de entre $80-90 \%$ en condiciones de saturación (Buytaert 2004) citado por (De Bievré 2008).

Los suelos de páramo son descritos como de alto contenido de materia orgánica (capa que supera algunas veces 1 metro de profundidad), baja densidad aparente y abiertos y porosos (De Bievré 2008). Los suelos del páramo también inciden en la provisión de otro SE: la captura de carbono. El suelo conjuntamente con las bajas temperaturas paramunas, hacen que este ecosistema genere una absorción neta de CO2 atmosférico (Procuraduría General de la Nación 2007), la cual es incluso superior (por hectárea) a la captura que realizan las selvas tropicales (Hofstede 2002).

La riqueza de las milenarias formas de apropiación física y simbólica de los páramos, que han significado el establecimiento de distintos modos de relacionamiento con este ecosistema, de diversas prácticas sociales para obtener acceso al páramo y lograr su control y de diferenciadas estrategias de aprovechamiento de sus recursos, sustentan la oferta de otra serie de SE de tipo cultural, los cuales son difícilmente reconocidos y poco investigados.

La apropiación física, ha significado la instalación de 
infraestructura, el uso potencial del páramo para actividades turísticas (Monasterio 2003), la realización de investigaciones y el establecimiento de actividades productivas en él. La apropiación simbólica del páramo por parte de los grupos indígenas, hizo que se considerara a los páramos como los generadores de la vida y la muerte, lugares sagrados, generadores del poder y la energía, donde los chamanes adquirían y ampliaban su conocimiento en alianza con las plantas de poder, los seres míticos, los dueños de la naturaleza silvestre y los espíritus tutelares de animales, territorios y las aguas (Portela 2000).

\section{Perdida y/o alteración de los SE en páramos}

La agricultura y la ganadería son la principal causa de la degradación de los ecosistemas de páramo. Estas actividades, que se desarrollaron en los páramos hace por lo menos 3.000 años A.P. (Hofstede et al. 1988) se han acelerado en los últimos 50 años, generando importantes cambios en el uso del suelo paramuno.

En el caso de la agricultura, el cultivo más dañino es la papa, la cual se da a alturas cada vez cercanas a los 4.000 m.s.n.m. Otras actividades que se realizan en los páramos colombianos pueden producir impactos considerables. De especial preocupación es la actividad minera, pues esta es una de las "locomotoras" del desarrollo nacional, lo que puede terminar estimulando la solicitud de concesiones mineras en zonas de páramo donde existen reservas de diversos minerales inexplotadas.

La conservación de la biodiversidad, es uno de los SE más afectados por la desaparición de la vegetación nativa del páramo. La agricultura a pequeña escala, remueve los frailejones y pequeños arbustos mediante el uso del machete para preparar la tierra; los grandes cultivadores han empezado a introducir maquinaria pesada para quitar la vegetación paramuna (Van der Hammen 2008). En la actividad ganadera, se usa fuego para impedir el crecimiento de la vegetación nativa y posibilitar la introducción de los pastos. Pero además, las plantas nativas no están preparadas para resistir la presencia de herbívoros como vacas, cabras y ovejas (Van der Hammen 2008). El peso y pastoreo de los animales fracciona las macollas.

La combinación de agricultura, ganadería y quema, conlleva la destrucción de madrigueras para mamíferos y de sitios de refugio para algunos anfibios, es decir se altera el hábitat natural de las especies, otro SE de gran importancia en los páramos. Con ello, se afectan rutas migratorias, nichos y hábitats de reproducción de especies, con lo cual algunas incluso se extinguen.
La regulación hídrica, se ve afectada por la erosión que genera el aumento de zonas del suelo desprovistas de vegetación y por su compactación originada en el pisoteo del ganado. Además, los cuerpos hídricos paramunos como lagunas, humedales y aguas subterráneas, se ven afectados en su cantidad y calidad (Instituto de Investigación de Recursos Biológicos Alexander von Humboldt 2007), básicamente porque la orina de los animales aumenta los niveles de nitrógeno en el suelo y los cuerpos hídricos del (Van der Hammen 2008).

La degradación de los páramos significa además la pérdida y/o alteración de los SE culturales de las comunidades ancestrales que sustentan su vida en estos ecosistemas. Para ellas, la desaparición del páramo significa la pérdida de referentes importantes para su diario vivir, como creencias, mitos, valores y conocimientos ancestrales.

\section{PAGO POR SERVICIOS AMBIENTALES}

\section{Antecedentes, definición y otras consideraciones}

El PSA se ha vuelto un tópico popular tanto a nivel académico como de diseñadores de políticas en los últimos 10 años (McElwee 2011), pues se consideran un enfoque novedoso para promover la conservación de los SE. Los PSA consisten en transferir un pago de quienes se benefician por un $\mathrm{SE}$ a quienes los generan o cuidan, básicamente los propietarios de la tierra donde se producen los SE (CAF \& The Nature Conservancy 2008). La racionalidad detrás de los PSA es que los propietarios de la tierra tienen pocos incentivos para proteger la naturaleza presente en su suelo, lo cual puede ser estimulado a través del pago de una compensación por parte de beneficiarios de los SE, la cual sea suficiente para motivar un uso del suelo ambientalmente más deseable.

Para Wunder (2005) los PSA se definen técnicamente como una transacción voluntaria y condicional, con al menos un vendedor, un comprador y un servicio ambiental bien definido, que es suministrado continuamente. Como mecanismo de compensación económica, los PSA intentan cambiar la lógica del que contamina paga a la lógica del que se beneficia (del SE) paga. Los propietarios del suelo pasan de ser vistos como contaminadores a proveedores de servicios.

La promoción del esquema PSA se da desde distintas disciplinas y por distintos motivos (McElwee 2011). Para ecologistas y biólogos, los PSA son una forma de reducir la degradación de la biodiversidad generando incentivos para disminuir la presión sobre la naturaleza. Para los economistas, los PSA son un esquema para mejorar la 
eficiencia en la distribución de los SE y corregir las externalidades; mientras quienes trabajan en el campo del desarrollo ven los PSA como una forma de generar incentivos orientados a los pobres y la promoción de bienes sociales.

Para tener un verdadero esquema de PSA se deben cumplir cuatro condiciones (Van Hecken \& Bastiaensen 2010). Primero, el mecanismo debe ser voluntario o sea que el proveedor del servicio decide si acepta la compensación o no. Segundo, el SE debe estar bien definido y ser entonces medible. Tercero, el PSA debe materializarse en la transferencia de recursos desde el comprador del SE a su proveedor. Finalmente, se de cumplir el criterio de condicionalidad que significa que el PSA se genera cuando se certifica y monitorea el cumplimiento de las acciones que garantizan el flujo del SE.

Los esquemas de PSA se han implementado en países desarrollados y en desarrollo (Wunder 2008). Algunos países como México, Costa Rica y Ecuador han incluido los PSA en sus políticas. Los tipos de PSA son muy diversos y variados, especialmente en América Latina.

Existen PSA que se diferencias según la fuente financiera: el pago lo realiza el gobierno con sus recursos o lo realizan los beneficiarios del SE (incluso es posible una mezcla de ambos). Otra forma de clasificación de los PSA es si se paga de acuerdo con el tamaño del área conservada o si se hace por un producto o SE específico; es posible clasificar los PSA de acuerdo a si la compensación se paga directamente al dueño de la tierra o si se compensa indirectamente, a través por ejemplo de la ejecución de obras, sin crear una relación proveedor-beneficiario (CAF \& The Nature Conservancy 2008).

\section{ESTUDIO DE CASO}

\section{PSA en Costa Rica}

Con base en el artículo publicado por Pagiola (2008), a continuación se presenta un ejemplo resumido del desarrollo de un esquema PSA en Costa Rica. Este país, fue pionero en implementar el esquema de PSA entre los países en desarrollo (Pagiola 2008). Allí se estableció un programa formal de PSA de aplicación nacional que empezó en 1997, a partir de la expedición de la Ley Forestal 7575 (1.996). Este programa reconoce cuatro servicios ambientales proporcionados por los ecosistemas forestales: i) mitigación de gases de efecto invernadero; ii) servicios hidrológicos, incluyendo agua para uso humano, agrícola y generación de energía; iii) conservación de la biodiversidad; y iv) provisión de belleza escénica para recreación y ecoturismo.
El Programa PSA no empezó de cero pues Costa Rica ya contaba con experiencias en pagos por reforestación y manejo de bosques, financiados con recursos del gobierno. El programa PSA es manejado por el Fondo Nacional de Financiamiento Forestal-FONAFIFO, entidad semiautónoma con estatus legal independiente, cuyo órgano directivo está conformado por tres representantes del gobierno (Ministerios de Ambiente y Agricultura y Sistema Bancario Nacional) y dos del sector privado.

El programa PSA está dirigido a propietarios privados de tierras que se encuentran por fuera del sistema de áreas protegidas del país y a quienes se les paga por tener plantaciones forestales y conservar los bosques. Se supone entonces que mantener la tierra en estos dos usos del suelo proporciona los cuatro servicios ecosistémicos que generan los ecosistemas forestales.

Los propietarios de la tierra interesados en participar en el programa PSA deben presentar un plan de manejo forestal sostenible preparado por un reforestador licenciado (denominado regente). El plan debe incluir el uso del suelo propuesto y suministrar información sobre topografía, clima, suelos, drenaje, uso actual del suelo, capacidad de carga respecto del uso actual del suelo; además del cronograma de monitoreo y planes para prevenir incendios forestales y la cacería ilegal.

Una vez el plan de manejo es aprobado, se firma un contrato entre el propietario y FONAFIFO. Así el propietario empieza a realizar las prácticas de uso del suelo por él ofrecidas y puede empezar a recibir sus respectivos pagos, los cuales se vuelven anuales. En el caso de las plantaciones forestales, el valor que se pagaba en 2006 era de US 816/ha, mientras que en el caso de la conservación del bosque se pagaban US 64/ha/año.

Tales pagos se hacen después que el regente ha verificado el cumplimiento de los acuerdos, mediante una visita. El valor de los pagos es exactamente igual para cualquier ecosistema forestal ubicado en cualquier lugar del país. El contrato de las plantaciones forestales se hacía a 10 años, aunque luego se estimulaba a extenderlo por 15 años más, a la vez que el contrato por conservación del bosque se firmaba por un periodo de 5 años.

El pago que reciben los propietarios por los servicios ecosistémicos que producen se percibe como bajo. Esto, porque el propietario debe descontar aproximadamente un $15 \%$ del pago para pagar el plan de manejo y el monitoreo al regente; además, de los gastos asociados a las inversiones que se deben realizar para garantizar la provisión de los SE. 
En relación con las fuentes de financiamiento del esquema PSA Costarricense, desde un inicio estaba planteado que los usuarios de los servicios ecosistémicos debían pagar por conservar un flujo constante de los mismos. Sin embargo, esto se ha logrado parcialmente. Las principales fuentes de financiamiento de los PSA son el impuesto a las ventas de combustibles fósiles que suman cerca de US 10 millones anuales y entre 2001 y 2006 un préstamo del Banco Mundial y una donación de GEF.

La mayoría de los beneficiarios de los cuatro servicios ecosistémicos que brindan los ecosistemas forestales, no están pagando por su provisión. En donde más se ha avanzado es en el pago del servicio de regulación hídrica, pues allí se han logrado vincular y firmar contratos con productores de energía hidroeléctrica y más recientemente embotelladoras, sistemas municipales de abastecimiento de agua, usuarios de sistemas de riego y hoteles.

Para la conservación de la biodiversidad no se ha logrado vincular a usuarios como la industria turística local y en el caso de la captura de carbono, Costa Rica ha buscado vender créditos de reducción de emisiones de carbono. En cuanto a la preservación de escenarios naturales no se han logrado firmar acuerdos con la industria turística ni con compañías de rafting.

El programa PSA de Costa Rica está bien establecido, es ampliamente conocido internamente y se percibe como un programa exitoso. Aun así, los usuarios de los SE todavía no contribuyen decididamente con la financiación del esquema.

\section{Análisis de la efectividad de los PSA para conservar los SE ofrecidos por los páramos}

Para analizar la efectividad de los PSA para conservar los SE ofrecidos por los páramos, se empezará por discutir las principales limitaciones del esquema y luego sus potencialidades.

Una primera limitación y quizá la más fuerte, es que se tiene aún un gran vacío en el conocimiento ecológico que permita caracterizar adecuadamente los SE que le dan sustento a los esquemas de PSA (Norgaard 2010). Esto quiere decir, que aún se desconoce una gran parte de los procesos y funciones ecológicas que dan origen a los SE.

En el caso de los páramos esta situación es aún más evidente, pues el ecosistema tiene un alto nivel de complejidad y una gran amalgama de procesos biofísicos sobre los cuales no se ha investigado de manera profunda. Se reconocen de manera evidente seis servicios ecosistémicos que brindan los páramos pero seguramente su riqueza biofísica y cultural implica que este ecosistema ofrece muchos más. Un ejemplo muy diciente de la aún incipiente investigación de SE brindados por los páramos es que para el servicio de regulación hídrica que es el más reconocido, De Bievré (2008) afirma que ni siquiera para este SE se ha logrado entender su dinámica de producción ecológica.

Una segunda limitación, fuertemente relacionada con la anterior, es que al no tener una definición exacta de los SE que ofrece el ecosistema analizado, es muy difícil determinar claramente la relación causal entre diversos usos del suelo y su impacto en la oferta de los SE. Así, si mediante el PSA se promueve un uso del suelo cualquiera para aumentar la oferta de un SE determinado, es casi imposible demostrar la eficiencia del esquema (Muradian 2010)], al carecer de la información requerida de la relación entre ambas variables.

Establecer en los páramos relaciones de tipo causal entre los usos del suelo que allí se dan y los SE proporcionados es casi imposible por dos factores. Primero, dado que el páramo es un ecosistema no lineal, pues ante perturbaciones similares (por ejemplo generadas por el uso agrícola), se pueden generar respuestas espaciales y temporales disimiles, que no sigan ningún tipo de patrón identificable. Segundo, porque entre los mismos ecosistemas de páramo existe una gran variedad, la cual dificulta incluso la determinación de sus límites, su definición y por tanto su caracterización.

Un tercer elemento limitante de los PSA tiene que ver con su lógica fundamentalmente economicista. El hecho que se proponga el pago de una compensación económica, puede resultar en que se ignoren reglas de tipo local y normas socialmente establecidas que son en muchos casos motivadores intrínsecos de la conservación de los SE (Muradian 2010). Las transacciones monetarias propuestas por el esquema PSA homogenizan los diferentes sistemas sociales y culturales y los reducen a la unidad monetaria.

En el caso de los páramos, las comunidades ancestrales indígenas y campesinas que se han relacionado de forma milenaria con este ecosistema, seguramente han desarrollado sistemas sociales y normas culturales (incluso prácticas religiosas), que los motivan a preservar este ecosistema y sus SE. La implementación de los PSA puede distorsionar los comportamientos de las comunidades, induciéndolas a adoptar prácticas foráneas que no sean las más sostenibles para la conservación de los SE, al cambiar su lógica de relacionamiento con el ecosistema. La dificultad en el monitoreo de los usos que los propietarios de la tierra le están dando al suelo, para conocer si están cumpliendo con el esquema acordado de preservación de los $\mathrm{SE}$, es el otro limitante del PSA. Generalmente el monitoreo de los cambios en el uso del suelo puede ser costoso, pues depende de 
imágenes satelitales o visitas de expertos para comprobar los cambios que se están presentando. Si el costo del monitoreo es muy alto y le significa una alta carga financiera al PSA, esta actividad tenderá a no llevarse a cabo o a hacerlo de manera esporádica afectando una de las características fundamentales del esquema: el criterio de condicionalidad.

Los páramos son ecosistemas que por su configuración biofísica generalmente son de difícil acceso y que por su extensión, pueden dificultar enormemente la realización de visitas de monitoreo. Incluso el uso de las imágenes satelitales, la aerofotografía y la cartografía pueden verse limitadas por la inexistencia de un sistema de producción de esta información con un estándar único, lo cual genera datos a diferentes escalas espaciales, temporales $y$ por tanto incompatibles.

La financiación de los PSA, particularmente cuando se tiene como objetivo que los beneficiarios de los SE contribuyan en gran medida con sus pagos, es bastante complicado. El caso de Costa Rica demuestra que aun cuando los beneficiarios valoran positivamente los PSA y se tiene una larga tradición en su implementación, no necesariamente se comprometen en su financiación. Esto se debe en parte a que existen SE de nivel global, como la captura de carbono y la regulación del clima para los cuales es difícil identificar quienes son los beneficiarios. Caso contrario sucede con la regulación hídrica, pues generalmente es posible identificar el sentido del flujo de agua en una cuenca y determinar cuáles son los usuarios del recurso y por tanto sus beneficiarios.

Los SE brindados por los páramos pueden ser tan variados y tener beneficiarios a escalas espaciales y temporales tan diversas, que seguramente identificarlos es problemático y más aún lograr su compromiso en la financiación del esquema.

Una limitación adicional de los PSA es que tienden a convertir en propietarios de los SE a quienes son los dueños de la tierra o a quienes custodian la provisión de los mismos. Esto puede tener el efecto perverso de privatizar los SE, los cuales son un bien público pues sus beneficios sociales deben tener una valoración mayor que sus beneficios privados.

En lo que respecta a las potencialidades del esquema de PSA, se tiene que en diversas experiencias, se ha logrado generar cambios positivos en el uso del suelo, que favorecen la provisión de ciertos SE. Así, se ha posibilitado que algunos proveedores de SE adopten el esquema y lo entiendan como una oportunidad para generar beneficios sociales y a la vez obtener una compensación por su esfuerzo ambiental. Es decir, que se ha introducido un cambio en la racionalidad de algunos agentes económicos, principalmente del sector
privado.Además, el resultado de los PSA es que en algunos ejemplos, se han incrementado las áreas en conservación, por fuera de los sistemas de áreas protegidas. Es clave también que en el establecimiento de los esquemas de PSA, los proveedores y beneficiarios de los SE establecen procesos de negociación que les permite entender la racionalidad y lógica con la que actúan otros actores sociales.

\section{CONCLUSIONES}

Los páramos son un ecosistema único que seguramente brindan una gran cantidad de SE, pero a los cuales solos se les reconoce una parte de ellos: la regulación hídrica, la captura de carbono, la provisión de hábitats, la conservación de la biodiversidad y los servicios culturales asociados a la apropiación física y cultural de estos ecosistemas.

Los esquemas de PSA que se vienen desarrollando en diversos países desarrollados y en desarrollo, son vistos como una oportunidad para la conservación de los SE que brindan ecosistemas estratégicos como los páramos. Se considera que el pago de una compensación a los proveedores de los SE por parte de quienes se benefician de los mismos, generaría un incentivo para que actividades como la agricultura y la ganadería cambien sus prácticas actuales y generen la conservación necesaria de estos SE.

Sin embargo, los PSA tienen muchas limitaciones que los convierten en un esquema poco efectivo para promover la conservación de los SE de los páramos. Sus limitaciones se asocian a la dificultad para definir y medir los SE que son la base del esquema PSA, pero también a la incapacidad para establecer relaciones causales entre los distintos usos del suelo y los cambios en los SE en los páramos. Adicionalmente, los PSA se fundamentan en incentivos únicamente económicos que dejan de lado normas sociales y culturales que también pueden contribuir a conservar los SE. El financiamiento es otro problema pues no muchos beneficiarios están dispuestos a pagar las compensaciones por el uso que hacen de los SE. Realizar el monitoreo de los compromisos adquiridos por proveedores de los SE, en cuanto a si están desarrollando los usos del suelo que contribuyen a conservar los SE es costoso y por tanto limita cumplir el criterio de condicionalidad que es fundamental en todo PSA. Finalmente, el esquema PSA tiene la tendencia a generar una apropiación privada de los SE, pues las compensaciones se dirigen a proveedores de ellos, que son generalmente los propietarios de la tierra. 


\section{REFERENCIAS}

BUYTAERT, W. 2004. The properties of the soils of the south Ecuadorian páramo and the impact of land use changes on their hydrology. Ph.D. thesis, Faculty of Agricultural and applied Biological Sciences, Katholieke Universiteit Leuven.

BUYTAERT, W. IÑIGUEZ, V. \& BIÈVRE, B. D. 2007. The effects of afforestation and cultivation on water yield in the Andean páramo. Forest Ecology and Management 251(1-2): 22-30.

CAF; GOBIERNO DE BOLIVIA \& THE NATURE CONSERVANCY. 2008. Taller regional: Conservando los servicios ambientales para la gente y la naturaleza. Bolivia.

CONGRESO NACIONAL DE PÁRAMOS. 2009. Conservación con equidad. Paipa, Boyacá.

COSTANZA, R. D'ARGE, R. DE GROOT, R. FARBER, S. GRASSO, M. HANNON, B. LIMBURG, K. NAEEM, S. O'NEIL, R. PARUELO, J. RASKIN, R.; SUTTON, P. \& VAN DEN BELT, M. 1997. The value of the world's ecosystem services and natural capital. Nature 387: 253-260.

DAILY, G. C. 1997. Introduction: what are ecosystem services. En: Daily, G.C. Nature's Services. Island Press. Washington D.C. 412 pp.

DE BIEVRÉ, B. 2008. Las amenazas a los servicios Ambientales hídricos que provee el páramo. En Procuraduría general de la Nación. Panorama y perspectivas sobre la gestión ambiental de los sistemas de páramo. Memorias.

EVALUACIÓN DE LOS ECOSISTEMAS DEL MILENIO. 2003.

FISHER, B. TURNER, K. \& MORLING, P. 2009. Defining and classifying ecosystem services for decisions making. Ecological Economics 68: 643-653.

GOMEZ-BAGGETHUM, E. DE GROOT, R. LOMAS, P. \& MONTES, C. 2010. The history of ecosystem services in economy theory and practice: from early notions to markets and payment schemes. Ecological Economics 69: 1209-1218.

HOFSTEDE, R. 2002. Los páramos andinos: su diversidad, sus habitantes, sus problemas y sus perspectivas. Un breve diagnóstico regional del estado de conservación de los páramos. En Congreso Mundial de Páramos, Tomo II.

HOFSTEDE, R. LIPS, J. M. \& JONGSMA, W. 1988. Geografía, ecología y forestación de la Sierra Alta del Ecuador. Ediciones
Abya-Yala, Quito. 242 pp.

INSTITUTO DE INVESTIGACIÓN DE RECURSOS BIOLÓGICOS ALEXANDER VON HUMBOLDT. 2007. Atlas de Páramos de Colombia. Bogotá, Colombia. 208 pp

MCELWEE, P. 2011. Payment for environmental service as neolioberal market-based forest conservation in Vietnam: panacea or a problem? Geoforum, artículo en prensa.

MINISTERIO DE MEDIO AMBIENTE. 2002. Páramos: Programa para el manejo sostenible y restauración de ecosistemas de la alta montaña colombiana. Bogotá.

MONASTERIO, M. 2003. Evolución y transformación de los páramos en la Cordillera de Mérida: paisajes naturales y culturales en Venezuela. En: MUJICA, E. (ed). Paisajes culturales en los Andes. Memoria narrativa, casos de estudio, conclusiones y recomendaciones de la Reunión de Expertos (Arequipa y Chivay, Perú, mayo de 1998). 99-109. Lima, Representación de UNESCO en Perú.

MURADIAN, R. CORVERA, E. PASCUAL, U. KOSOY, N. \&MAY, P. 2010. Reconciling theory and practice: An alternative conceptual framework for understanding payment for environmental service. Ecological Economics 69: 1202-1208.

NORGAARD, R. 2010. Ecosystem services: from eye-opening metaphor to complexity blinder. Ecological Economics 69 (6): 1219-1227.

OJEDA, D. PINTO, M. CARDONA, M CUELLAR, S. CRUZ, L. DE LA TORRE, J. CASTAÑEDA, C. BARRERA, \& GONZALES, C. 2001. Ecosistemas. En LEYVA, P. (ed). El medio ambiente en Colombia. IDEAM, Bogotá.

PAGIOLA, S. 2008. Payment for environmental services in Costa Rica. Ecological Economics 65: 712-724.

PETERSON, M. HALL, D. FELDPAUSCH-PARKER, A. \& RAI, P. 2010. Obscuring ecosystem functions with the application of the ecosystem services concept. Conservation Biology 24(1): 113-119.

PORTELA, H. 2000. El pensamiento de las aguas de las montañas. Coconucos, Guambianos, Paeces, Yanaconas. Serie Estudios Sociales. Universidad del Cauca, Popayán.

PROCURADURÍA GENERAL DE LA NACIÓN. 2007. Informe preventivo: situación de los páramos en Colombia frente a la actividad antrópica y el cambio climático. Bogotá. 
RANGEL, O. 2002. Biodiversidad en la región del páramo: con especial referencia a Colombia. Congreso Mundial de Páramos. MMA, CAR, IDEAM y Colombia, C. Paipa. I: 168200.

RANGEL-CH, J. 2008. La función natural de la alta montaña en Colombia: servicios ambientales. En Procuraduría general de la Nación. Panorama y perspectivas sobre la gestión ambiental de los sistemas de páramo. Memorias.

REYES, P. 1996. El páramo un ecosistema de alta montaña. En Biblioteca Virtual banco de la República, página web: http://www.banrepcultural.org/blaavirtual/faunayflora/pa ramo/indice.htm, acceso octubre 5 de 2010.

UICN, UNIÓN MUNDIAL PARA LA NATURALEZA (Global Peakland Initiative; y, Ecociencia. 2003. Los Paramos del mundo. Quito, Ecuador.

VAN DER HAMMEN, T. 2008. De la destrucción a la conservación. En: Procuraduría general de la Nación (2008). Panorama y perspectivas sobre la gestión ambiental de los sistemas de páramo. Memorias.

VAN HECKEN, G. \& BASTIAENSEN, J. 2010. Payment for ecosystem services: justified or not? A political view. Environmental Science \& Policy 13: 785-792.

WUNDER, S. 2005. Payment for environmental services: some nuts and bolds. CIFOR Ocassional Papper No 42.

WUNDER, S. ENGEL, S. \& PAGUIOLA, S. 2008. Taking stock: A comparative analysis of payment for environmental services programs in developed and developing countries. Ecological Economics 65: 834-852. 